\title{
ENERGY CONSERVATION FOR THE WEAK SOLUTIONS OF THE COMPRESSIBLE NAVIER-STOKES EQUATIONS
}

\author{
CHENG YU
}

\begin{abstract}
In this paper, we prove the energy conservation for the weak solutions of the compressible Navier-Stokes equations for any time $t>0$, under certain conditions. The results hold for the renormalized solutions of the equations with constant viscosities, as well as the weak solutions of the equations with degenerate viscosity. Our conditions do not depend on the dimensions. The energy may conserve on the vacuum for the compressible Navier-Stokes equations with constant viscosities. Our results are the first ones on the energy conservation for the weak solutions of the compressible Navier-Stokes equations.
\end{abstract}

\section{INTRODUCTION}

This paper deals with the energy conservation for the weak solutions of the compressible Navier-Stokes equations, namely

$$
\begin{aligned}
& \rho_{t}+\operatorname{div}(\rho \mathbf{u})=0 \\
& (\rho \mathbf{u})_{t}+\operatorname{div}(\rho \mathbf{u} \otimes \mathbf{u})+\nabla P-2 \mu \Delta \mathbf{u}-\lambda \nabla \operatorname{div} \mathbf{u}=0,
\end{aligned}
$$

as well as the following equations with degenerate viscosity

$$
\begin{aligned}
& \rho_{t}+\operatorname{div}(\rho \mathbf{u})=0 \\
& (\rho \mathbf{u})_{t}+\operatorname{div}(\rho \mathbf{u} \otimes \mathbf{u})+\nabla P-2 \nu \operatorname{div}(\rho \mathbb{D} \mathbf{u})=0,
\end{aligned}
$$

respectively with initial data

$$
\left.\rho\right|_{t=0}=\rho_{0}(x),\left.\quad \rho \mathbf{u}\right|_{t=0}=\mathbf{m}_{0}(x)=\rho_{0} \mathbf{u}_{0},
$$

where $P=\rho^{\gamma}, \gamma>1$, denotes the pressure, $\rho$ is the density of fluid, $\mathbf{u}$ stands for the velocity of fluid, $\mathbb{D} \mathbf{u}=\frac{1}{2}\left[\nabla \mathbf{u}+\nabla^{T} \mathbf{u}\right]$ is the strain tensor. The viscosity coefficients $\mu, \lambda$ satisfies $\mu>0,2 \mu+N \lambda \geq 0$. For the sake of simplicity we will consider the case of bounded domain with periodic boundary conditions in $\mathbb{R}^{N}$, namely $\Omega=\mathbb{T}^{N}, N=2,3$. Here we define $\mathbf{u}_{0}=0$ on the set $\left\{x \mid \rho_{0}(x)=0\right\}$. Without loss of generality, we will fix $2 \nu=1$ in (1.2) from now on.

As we all known, the global existence of weak solution to (1.1), (1.3) was established in [11, 12, 19] for any $\gamma>\frac{N}{2}$. The weak solution of (1.2)-(1.3) was studied in [1, 2, 3, 4, 18, 21, 24]. The purpose of this paper is to provide the sufficient conditions for the energy conservation of the weak solutions of (1.2) first, then extend our result to the weak solutions of (1.1).

Date: April 30, 2018.

Key words and phrases. Energy conservation, Navier-Stokes equations, degenerate viscosity, weak solution. 
A weak solution $(\rho, \mathbf{u})$ to (1.2)-(1.3) constructed in 24] satisfies the following energy inequality

$$
\int_{\Omega}\left(\frac{1}{2} \rho|\mathbf{u}|^{2}+\frac{\rho^{\gamma}}{\gamma-1}\right) d x+\int_{0}^{T} \int_{\Omega} \rho|\mathbb{D} \mathbf{u}|^{2} d x d t \leq \int_{\Omega}\left(\frac{1}{2} \rho_{0}\left|\mathbf{u}_{0}\right|^{2}+\frac{\rho_{0}^{\gamma}}{\gamma-1}\right) d x,
$$

even if $\rho \geq \underline{\rho}>0$. A natural question to ask is when a weak solution $(\rho, \mathbf{u})$ of the compressible Navier-Stokes equations (1.2)-(1.3) satisfies, not only (1.4), but the stronger version

$$
\int_{\Omega}\left(\frac{1}{2} \rho|\mathbf{u}|^{2}+\frac{\rho^{\gamma}}{\gamma-1}\right) d x+\int_{0}^{T} \int_{\Omega} \rho|\mathbb{D} \mathbf{u}|^{2} d x d t=\int_{\Omega}\left(\frac{1}{2} \rho_{0}\left|\mathbf{u}_{0}\right|^{2}+\frac{\rho_{0}^{\gamma}}{\gamma-1}\right) d x .
$$

It is well known that if a solution is smooth enough, then it conserves the energy. Thus, our question is connected to the regularity of weak solutions. However, the regularity of global weak solutions of (1.2) remains yet mostly open. Naturally, of particular interest is the question: how badly behaved $(\rho, \mathbf{u})$ can be so that it keeps energy conservation? In mathematics, how much regularity is needed for a weak solution to ensure energy equality (1.5)? We can ask the same question for the weak solutions of equation (1.1). The main contribution of our paper is to provide the first result on this topic of the compressible Navier-Stokes equations for any $t>0$.

As we mentioned before, the question to ask is how much regularity is necessary to conserve the energy. In the context of the incompressible Navier-Stokes equations, the pioneer study was done by Serrin [22]. He has proved that a weak solution $\mathbf{u}$ conserves its energy globally, provided $\mathbf{u} \in L^{p}\left(0, T ; L^{q}(\Omega)\right)$, where

$$
\frac{2}{p}+\frac{N}{q} \leq 1
$$

where $N$ is the dimension. Later, Shinbrot [23] proved the same conclusion if

$$
\frac{2}{p}+\frac{2}{q} \leq 1
$$

and $q \geq 4$. Meanwhile, in the context of incompressible Euler equations, this question is linked to a famous conjecture of Onsager [15]: energy should be conserved if the solution is Holder continuous with exponent greater than $1 / 3$, while solutions with less regularity possibly dissipate energy. The first part was solved by [7, 8, 10] while significant progress has recently been made on the second part [5, 6]. Very recently, Isett in [14] solved the second part of Onsager conjecture. Feireisl-Gwiazda-Swierczewska-Gwiazda-Wiedemann [13. gave sufficient conditions on the regularity of solutions to the inhomogeneous incompressible Euler and the compressible isentropic Euler systems in order for the energy to be conserved in the distribution sense. Leslie-Shvydkoy [16] showed the energy balance relation for density dependent incompressible Navier-Stokes holds for weak solutions if the velocity, density and pressure belong to a range Besov spaces of smoothness $\frac{1}{3}$. To our best knowledge, there is no available result for the weak solutions of the compressible Navier-Stokes equations.

The sufficient conditions for the energy conservation are addressed in this paper. Our approach relies on the idea of Vasseur-Yu [24] and $\mathrm{Yu}$ [25]. Compared to the incompressible Navier-Stokes equations, we are not able to deduce that $\mathbf{u}(t, x)$ is continuous at time 
$t=0$. Our alternative way for the compressible Navier-Stokes equations is to gain the continuity of $\rho(t)$ and $(\sqrt{\rho} \mathbf{u})(t)$ in the strong topology at $t=0$. To this end, we need $\nabla \sqrt{\rho} \in L^{\infty}\left(0, T ; L^{2}(\Omega)\right)$. Lucky, BD entropy (see [1, 2, 3]) gives us such an estimate on density for the degenerate compressible Navier-Stokes equations. However, we need additional condition on the density to study the weak solutions of (1.1). Meanwhile, it is crucial to rely on Lemma 2.3 to handle the nonlinear compositions $(\rho \mathbf{u})_{t}$ and $\rho \mathbf{u} \otimes \mathbf{u}$. Here we have to mention that our approach allows us to handle vacuum states for the weak solutions of (1.1).

The question addressed here is motivated by the fact that the energy conservation is fundamental both in the physical theory as well as in the mathematical study of the fluid dynamics. It is natural, therefore, to seek a rigorous theory which accommodates this question. The results of this paper effectively achieve this goal by providing a certain condition for the weak solution, for any $t>0$. To address our main result, we now give a precise definition and discussion of our weak solutions $(\rho, \mathbf{u})$ to the initial value problem (1.2)-(1.3) in the following sense.

Definition 1.1. The $(\rho, \mathbf{u})$ is called a global weak solution to (1.2)-(1.3), if $(\rho, \mathbf{u})$ satisfies the following ones, for any $t \in[0, T]$,

- (1.2) holds in $\left.\mathcal{D}^{\prime}((0, T) \times \Omega)\right)$ and the following is satisfied $\rho \geq 0, \quad \rho \in L^{\infty}\left([0, T] ; L^{\gamma}(\Omega)\right)$, $\nabla \rho^{\frac{\gamma}{2}} \in L^{2}\left(0, T ; L^{2}(\Omega)\right), \quad \nabla \sqrt{\rho} \in L^{\infty}\left(0, T ; L^{2}(\Omega)\right)$, $\sqrt{\rho} \mathbf{u} \in L^{\infty}\left(0, T ; L^{2}(\Omega)\right), \quad \sqrt{\rho \nabla} \mathbf{u} \in L^{2}\left(0, T ; L^{2}(\Omega)\right)$,

- (1.3) holds in $\mathcal{D}^{\prime}(\Omega)$.

The global existence of weak solution to (1.2) under the above definition was proved in [24]. The energy inequality (1.4) holds for almost every $t \in[0, T]$ if the density is bounded away from zero. Here we state the following result on the energy conservation for the weak solution.

Theorem 1.1. Let $(\rho, \mathbf{u})$ be a weak solution of (1.2)-(1.3) in the sense of Definition 1.1. Moreover, if

$$
\begin{gathered}
0<\underline{\rho} \leq \rho(t, x) \leq \bar{\rho}<\infty \\
\mathbf{u} \in L^{p}\left(0, T ; L^{q}(\Omega)\right) \quad \text { for any } \frac{1}{p}+\frac{1}{q} \leq \frac{5}{12} \text { with } q \geq 6,
\end{gathered}
$$

and

$$
\sqrt{\rho_{0}} \mathbf{u}_{0} \in L^{4}(\Omega)
$$

then such a weak solution $(\rho, \mathbf{u})$ satisfies (1.5) for any $t \in[0, T]$.

Remark 1.1. The condition (1.8) could be replaced by (1.11). We will give detail on this issue at the end of this paper.

Remark 1.2. On the incompressible Navier-Stokes equations, Shinbrot [23] has shown that a weak solution can remain its energy if

$$
\mathbf{u} \in L^{p}\left(0, T ; L^{q}(\Omega)\right)
$$


for any $\frac{1}{p}+\frac{1}{q} \leq \frac{1}{2}$ with $q \geq 4$. A new proof of Shinbrot's result is given in [25]. Our condition (1.10) for the compressible version is slightly stronger than the incompressible ones. Because we have to rely on the commutator lemma (Lemma 2.3) to handle the terms $(\rho \mathbf{u})_{t}$ and $\operatorname{div}(\rho \mathbf{u} \otimes \mathbf{u})$, which needs additional regularity on $\mathbf{u}$.

Next we extend Theorem 1.1 to the weak solution of (1.1) in the following theorem. The global existence of renormalized weak solutions to (1.1) was established in [19, 11, 12 for any $\gamma>\frac{N}{2}$. In particular, we need additional restriction (1.9) for density, which allows us to have the continuity of $\rho(t)$ and $(\sqrt{\rho} \mathbf{u})(t)$ in the strong topology at $t=0$. Meanwhile, $\mathbf{u}$ is uniformly bounded in $L^{2}\left(0, T ; H^{1}(\Omega)\right)$ for the viscosity constants case. Thus, our approach allows us to handle vacuum states. With the restriction (1.9) in mind, we can modify the proof of Theorem 1.1 to show the following result. One slight difference is to show $W=0$ in (2.26) for getting the continuity of $\rho(t)$ and $(\sqrt{\rho} \mathbf{u})(t)$ in the strong topology at $t=0$. We will give the detail on this point at the end of this paper.

Theorem 1.2. Let $(\rho, \mathbf{u})$ be a weak solution of (1.1), (1.3) in the sense of [11, 20]. Moreover, if

$$
\begin{gathered}
0 \leq \rho(t, x) \leq \bar{\rho}<\infty, \quad \text { and } \nabla \sqrt{\rho} \in L^{\infty}\left(0, T ; L^{2}(\Omega)\right), \\
\mathbf{u} \in L^{p}\left(0, T ; L^{q}(\Omega)\right) \quad \text { for any } \frac{1}{p}+\frac{1}{q} \leq \frac{5}{12}, \text { and } q \geq 6,
\end{gathered}
$$

and

$$
\mathbf{u}_{0} \in L^{k}(\Omega), \frac{1}{k}+\frac{1}{q} \leq \frac{1}{2},
$$

then such a weak solution $(\rho, \mathbf{u})$ satisfies

$$
\begin{aligned}
\int_{\Omega}\left(\frac{1}{2} \rho|\mathbf{u}|^{2}+\right. & \left.\frac{\rho^{\gamma}}{\gamma-1}\right) d x+2 \mu \int_{0}^{T} \int_{\Omega}|\nabla \mathbf{u}|^{2} d x d t \\
& +\lambda \int_{0}^{T} \int_{\Omega}|\operatorname{div} \mathbf{u}|^{2} d x d t=\int_{\Omega}\left(\frac{1}{2} \rho_{0}\left|\mathbf{u}_{0}\right|^{2}+\frac{\rho_{0}^{\gamma}}{\gamma-1}\right) d x
\end{aligned}
$$

for any $t \in[0, T]$.

Remark 1.3. An interesting point is that the density may vanish in (1.9). Thus, Theorem 1.2 indicates that the energy may conserve even on the vacuum.

\section{Proof of MAIN RESUlts}

The main object of this section is to prove our main results, including Theorem 1.1 and Theorem 1.2. We devote subsection 2.1 to a proof of Theorem 1.1, and subsection 2.2 to a proof of Theorem 1.2. 
2.1. Proof of Theorem 1.1. Note that, for any weak solution $(\rho, \mathbf{u})$, with condition (1.6), it satisfies

$$
\|\mathbf{u}\|_{L^{\infty}\left(0, T ; L^{2}(\Omega)\right)} \leq C<\infty, \quad\|\nabla \mathbf{u}\|_{L^{2}\left(0, T ; L^{2}(\Omega)\right)} \leq C<\infty .
$$

Now, let us to give an estimate on $\rho_{t}$ in the following lemma.

Lemma 2.1. For any weak solution $(\rho, \mathbf{u})$ in the sense of Definition 1.1 with additional conditions (1.6) $-(1.10)$, then $\rho_{t}$ is bounded in $L^{p}\left(0, T ; L^{\frac{2 q}{q+2}}(\Omega)\right)+L^{2}\left(0, T ; L^{2}(\Omega)\right)$. In particular, $\rho_{t}$ is bounded in $L^{2}\left(0, T ; L^{\frac{2 q}{q+2}}(\Omega)\right)$ if $p \geq 2$.

Proof. Note that

$$
\rho_{t}=-2 \sqrt{\rho} \mathbf{u} \cdot \nabla \sqrt{\rho}-\sqrt{\rho} \sqrt{\rho} \operatorname{div} \mathbf{u},
$$

consequently $\rho_{t}$ is bounded in $L^{p}\left(0, T ; L^{\frac{2 q}{q+2}}(\Omega)\right)+L^{2}\left(0, T ; L^{2}(\Omega)\right)$, thanks to the estimates in definition 1.1, (1.6) and (1.10).

Remark 2.1. For any weak solution $(\rho, \mathbf{u})$ satisfied B-D entropy [1, 2, 3], $\nabla \sqrt{\rho}$ is bounded in $L^{\infty}\left(0, T ; L^{2}(\Omega)\right)$. Thus, Lemma 2.1 gives us that $\rho_{t}$ is locally integrable. It is crucial to have the estimate of $\nabla \sqrt{\rho}$ for making commutator estimates.

Here we make use of $L^{p}-L^{q}$ inequality to deduce the following lemma directly.

Lemma 2.2. If $\mathbf{u} \in L^{\infty}\left(0, T ; L^{2}(\Omega)\right) \cap L^{p}\left(0, T ; L^{q}(\Omega)\right)$, then there exists some $\alpha \in(0,1)$ such that $\mathbf{u} \in L^{r}\left(0, T ; L^{s}(\Omega)\right)$, for any

$$
\frac{1}{r}=\frac{1-\alpha}{p}
$$

and

$$
\frac{1}{s}=\frac{\alpha}{2}+\frac{1-\alpha}{q}
$$

Now, let us to define

$$
\bar{f}(t, x)=\eta_{\varepsilon} * f(t, x), \quad t>\varepsilon,
$$

where $\eta_{\varepsilon}=\frac{1}{\varepsilon^{N+1}} \eta\left(\frac{t}{\varepsilon}, \frac{x}{\varepsilon}\right)$, and $\eta(t, x) \geq 0$ is a smooth even function compactly supported in the space time ball of radius 1 , and with integral equal to 1.

The following lemma is crucial in this current paper, which was proved by Lions in [19]. Here we adopt the following statement of [17].

Lemma 2.3. Let $\partial$ be a partial derivative in space or time. Let $f, \partial f \in L^{p}\left(\mathbb{R}^{+} \times \Omega\right)$, $g \in L^{q}\left(\mathbb{R}^{+} \times \Omega\right)$ with $1 \leq p, q \leq \infty$, and $\frac{1}{p}+\frac{1}{q} \leq 1$. Then, we have

$$
\|\overline{\partial(f g)}-\partial(f \bar{g})\|_{L^{r}\left(\mathbb{R}^{+} \times \Omega\right)} \leq C\|\partial f\|_{L^{p}\left(\mathbb{R}^{+} \times \Omega\right)}\|g\|_{L^{q}\left(\mathbb{R}^{+} \times \Omega\right)}
$$

for some constant $C>0$ independent of $\varepsilon, f$ and $g$, and with $\frac{1}{r}=\frac{1}{p}+\frac{1}{q}$. In addition,

$$
\overline{\partial(f g)}-\partial(f \bar{g}) \rightarrow 0 \quad \text { in } L^{r}\left(\mathbb{R}^{+} \times \Omega\right)
$$

as $\varepsilon \rightarrow 0$ if $r<\infty$. 
With Lemma 2.1 Lemma 2.3 in hand, we are ready to show Theorem 1.1. Here we introduce a new function $\Phi=\overline{\psi(t) \overline{\mathbf{u}}}$. Note that $\psi(t) \in \mathfrak{D}(0,+\infty)$ is a test function, where $\mathfrak{D}(0,+\infty)$ is a class of all smooth compactly supported functions in $(0,+\infty)$. In particular, this function vanishes close $t=0$. However, it is needed to extend the result for $\psi(t) \in \mathfrak{D}(-1,+\infty)$.

Note that, $\psi(t)$ is compactly supported in $(0, \infty)$. $\Phi$ is well defined on $(0, \infty)$ for $\varepsilon$ small enough. Using it to test the second equation in (1.2), one obtains

$$
\int_{0}^{T} \int_{\Omega} \bar{\Phi}\left((\rho \mathbf{u})_{t}+\operatorname{div}(\rho \mathbf{u} \otimes \mathbf{u})+\nabla \rho^{\gamma}-\operatorname{div}(\rho \mathbb{D} \mathbf{u})\right) d x d t=0
$$

which in turn yields

$$
\int_{0}^{T} \int_{\Omega} \psi(t) \overline{\mathbf{u}} \overline{\left.(\rho \mathbf{u})_{t}+\operatorname{div}(\rho \mathbf{u} \otimes \mathbf{u})+\nabla \rho^{\gamma}-\operatorname{div}(\rho \mathbb{D} \mathbf{u})\right)} d x d t=0
$$

where we used a fact $\eta(-t,-x)=\eta(t, x)$.

The first term in (2.2) shows that

$$
\begin{aligned}
\int_{0}^{T} \int_{\Omega} \psi(t) \overline{\mathbf{u}} \overline{(\rho \mathbf{u})_{t}} d x & =\int_{0}^{T} \int_{\Omega} \psi(t)\left(\overline{(\rho \mathbf{u})_{t}}-(\rho \overline{\mathbf{u}})_{t}\right) \overline{\mathbf{u}} d x d t+\int_{0}^{T} \int_{\Omega} \psi(t)(\rho \overline{\mathbf{u}})_{t} \overline{\mathbf{u}} d x d t \\
& =A+\int_{0}^{T} \int_{\Omega} \psi(t) \rho \partial_{t} \frac{|\overline{\mathbf{u}}|^{2}}{2} d x d t+\int_{0}^{T} \int_{\Omega} \psi(t) \rho_{t}|\overline{\mathbf{u}}|^{2} d x d t .
\end{aligned}
$$

Similarly, the second term in (2.2) gives us

$$
\begin{aligned}
& \int_{0}^{T} \int_{\Omega} \psi(t) \overline{\mathbf{u}} \overline{\operatorname{div}(\rho \mathbf{u} \otimes \mathbf{u})} d x d t=\int_{0}^{T} \int_{\Omega} \psi(t)(\operatorname{div}(\overline{\rho \mathbf{u} \otimes \mathbf{u})}-\operatorname{div}(\rho \mathbf{u} \otimes \overline{\mathbf{u}})) \overline{\mathbf{u}} d x d t \\
& +\int_{0}^{T} \int_{\Omega} \psi(t) \operatorname{div}(\rho \mathbf{u} \otimes \overline{\mathbf{u}}) \overline{\mathbf{u}} d x d t \\
& =B+\int_{0}^{T} \int_{\Omega} \psi(t) \rho \mathbf{u} \cdot \nabla \frac{|\overline{\mathbf{u}}|^{2}}{2} d x+\int_{0}^{T} \int_{\Omega} \psi(t) \operatorname{div}(\rho \mathbf{u})|\overline{\mathbf{u}}|^{2} d x d t \\
& =B+\int_{0}^{T} \int_{\Omega} \psi(t) \rho_{t} \frac{|\overline{\mathbf{u}}|^{2}}{2} d x+\int_{0}^{T} \int_{\Omega} \psi(t) \operatorname{div}(\rho \mathbf{u})|\overline{\mathbf{u}}|^{2} d x d t .
\end{aligned}
$$

Thanks to Lemma 2.1, the last term of the right-hand side in (2.3) and the second term of the right-hand side in (2.4) are well defined. Combining (2.3)-(2.4), the first two terms in (2.2) are given by

$$
\int_{0}^{T} \int_{\Omega} \psi(t)\left(\frac{1}{2} \rho|\overline{\mathbf{u}}|^{2}\right)_{t} d x d t+A+B
$$


Next, the last term in (2.2) gives us

$$
\begin{aligned}
\int_{0}^{T} \int_{\Omega} \psi(t) \overline{\operatorname{div}(\rho \mathbb{D} \mathbf{u})} \overline{\mathbf{u}} d x d t & =\int_{0}^{T} \int_{\Omega} \psi(t)(\overline{\operatorname{div}(\rho \mathbb{D} \mathbf{u})}-\operatorname{div}(\rho \overline{\mathbb{D} \mathbf{u}})) \overline{\mathbf{u}} d x d t \\
& +\int_{0}^{T} \int_{\Omega} \psi(t) \operatorname{div}(\rho \overline{\mathbb{D} \mathbf{u}}) \overline{\mathbf{u}} d x d t \\
& =D-\int_{0}^{T} \int_{\Omega} \psi(t) \rho|\overline{\mathbb{D} \mathbf{u}}|^{2} d x d t
\end{aligned}
$$

We estimate the third term in (2.2) as follows

$$
\begin{aligned}
& \int_{0}^{T} \int_{\Omega} \psi(t) \overline{\nabla \rho^{\gamma}} \overline{\mathbf{u}} d x d t=\frac{\gamma}{\gamma-1} \int_{0}^{T} \int_{\Omega} \psi(t) \overline{\rho \nabla \rho^{\gamma-1}} \overline{\mathbf{u}} d x d t \\
& =\frac{\gamma}{\gamma-1} \int_{0}^{T} \int_{\Omega} \psi(t)\left(\overline{\rho \nabla \rho^{\gamma-1}}-\rho \overline{\nabla \rho^{\gamma-1}}\right) \overline{\mathbf{u}} d x d t+\frac{\gamma}{\gamma-1} \int_{0}^{T} \int_{\Omega} \psi(t) \rho \overline{\nabla \rho^{\gamma-1}} \overline{\mathbf{u}} d x d t \\
& =E-\frac{\gamma}{\gamma-1} \int_{0}^{T} \int_{\Omega} \psi(t) \operatorname{div}(\rho \overline{\mathbf{u}}) \overline{\rho^{\gamma-1}} d x d t .
\end{aligned}
$$

The second term in the last equality of (2.7) shows that

$$
\begin{aligned}
& \frac{\gamma}{\gamma-1} \int_{0}^{T} \int_{\Omega} \psi(t) \operatorname{div}(\rho \overline{\mathbf{u}}) \overline{\rho^{\gamma-1}} d x d t \\
& =\frac{\gamma}{\gamma-1} \int_{0}^{T} \int_{\Omega} \psi(t)(\operatorname{div}(\rho \overline{\mathbf{u}})-\overline{\operatorname{div}(\rho \mathbf{u})}) \overline{\rho^{\gamma-1}} d x d t+\frac{\gamma}{\gamma-1} \int_{0}^{T} \int_{\Omega} \psi(t) \overline{\operatorname{div}(\rho \mathbf{u})} \overline{\rho^{\gamma-1}} d x d t \\
& =E_{21}-\frac{\gamma}{\gamma-1} \int_{0}^{T} \int_{\Omega} \psi(t) \bar{\rho}_{t} \overline{\rho^{\gamma-1}} d x d t .
\end{aligned}
$$

Meanwhile, the last term in (2.8) is as follows

$$
\begin{aligned}
& \frac{\gamma}{\gamma-1} \int_{0}^{T} \int_{\Omega} \psi(t) \bar{\rho}_{t} \overline{\rho^{\gamma-1}} d x d t \\
& =\frac{\gamma}{\gamma-1} \int_{0}^{T} \int_{\Omega} \psi(t)\left(\bar{\rho}_{t}-\rho_{t}\right) \overline{\rho^{\gamma-1}} d x d t+\frac{\gamma}{\gamma-1} \int_{0}^{T} \int_{\Omega} \psi(t)\left(\rho_{t} \overline{\rho^{\gamma-1}}-\rho_{t} \rho^{\gamma-1}\right) d x d t \\
& +\frac{\gamma}{\gamma-1} \int_{0}^{T} \int_{\Omega} \psi(t) \rho_{t} \rho^{\gamma-1} d x d t \\
& =E_{31}+E_{32}+\frac{1}{\gamma-1} \int_{0}^{T} \int_{\Omega} \psi(t)\left(\rho^{\gamma}\right)_{t} d x d t
\end{aligned}
$$

Thanks to (2.5) $-(2.9),(2.2)$ gives us

$$
\begin{aligned}
& \int_{0}^{T} \int_{\Omega} \psi(t)\left(\frac{1}{2} \rho|\overline{\mathbf{u}}|^{2}+\frac{\rho^{\gamma}}{\gamma-1}\right)_{t} d x d t \\
& +\int_{0}^{T} \int_{\Omega} \psi(t) \rho|\overline{\mathbb{D} \mathbf{u}}|^{2} d x d t+A+B-D+E-E_{21}+E_{31}+E_{32}=0 .
\end{aligned}
$$


This yields,

$$
\begin{aligned}
-\int_{0}^{T} \int_{\Omega} \psi_{t}\left(\frac{1}{2} \rho|\overline{\mathbf{u}}|^{2}+\frac{\overline{\rho^{\gamma}}}{\gamma-1}\right) d x d t & \\
& +\int_{0}^{T} \int_{\Omega} \psi(t) \rho|\overline{\mathbb{D} \mathbf{u}}|^{2} d x d t+R_{\varepsilon}(t, x)=0
\end{aligned}
$$

where $R_{\varepsilon}(t, x)=A+B-D+E-E_{21}+E_{31}+E_{32}$.

Note that (1.6) and (1.10), one obtains

$$
\int_{0}^{T} \int_{\Omega} \frac{1}{2} \rho|\overline{\mathbf{u}}|^{2} \psi_{t} d x d t \rightarrow \int_{0}^{T} \int_{\Omega} \frac{1}{2} \rho|\mathbf{u}|^{2} \psi_{t} d x d t \text { as } \varepsilon \rightarrow 0 .
$$

Thanks to (1.6) and (2.1), we find that, for any $\varepsilon$ tends to zero,

$$
\int_{0}^{T} \int_{\Omega} \psi(t) \rho|\overline{\mathbb{D} \mathbf{u}}|^{2} d x d t \rightarrow \int_{0}^{T} \int_{\Omega} \psi(t) \rho|\mathbb{D} \mathbf{u}|^{2} d x d t .
$$

The next step is to make use of Lemma 2.3 to prove

$$
R_{\varepsilon}(t, x) \rightarrow 0
$$

as $\varepsilon$ goes to zero. First, we assume that $\mathbf{u}$ is bounded in $L^{r}\left(0, T ; L^{s}(\Omega)\right)$. We will improve this restriction later. Under this restriction, by Lemma 2.1, $\rho_{t}$ is uniformly bounded in $L^{r}\left(0, T ; L^{\frac{2 s}{s+2}}(\Omega)\right)+L^{2}\left(0, T ; L^{2}(\Omega)\right)$. Thus, Lemma 2.3 gives us

$$
\begin{aligned}
|A| & \left.\leq\|\psi(t)\|_{L^{\infty}(0, T)} \int_{0}^{T} \int_{\Omega} \mid \overline{\mathbf{u}} \overline{(\rho \mathbf{u})_{t}}-(\rho \overline{\mathbf{u}})_{t}\right] \mid d x d t \\
& \leq C\|\psi(t)\|_{L^{\infty}(0, T)}\left\|\rho_{t}\right\|_{L^{2}\left(0, T ; L^{\frac{2 s}{2+s}}(\Omega)\right)}\|\mathbf{u}\|_{L^{r}\left(0, T ; L^{s}(\Omega)\right)}^{2},
\end{aligned}
$$

for any $r \geq 4$ and $s \geq 6$.

Meanwhile, for any $\mathbf{u} \in L^{p}\left(0, T ; L^{q}(\Omega)\right)$, by Lemma 2.2 , one obtains that for $0<\alpha<1$,

$$
\frac{1-\alpha}{p}=\frac{1}{r}, \quad \text { and } \frac{\alpha}{2}+\frac{1-\alpha}{q}=\frac{1}{s}
$$

with $q \geq 6$. This gives us, for any $0<\alpha<1$

$$
\left(\frac{1}{p}+\frac{1}{q}\right)(1-\alpha) \leq\left(\frac{1}{p}+\frac{1}{q}\right)(1-\alpha)+\frac{\alpha}{12} \leq \frac{5}{12}(1-\alpha)
$$

and thus

$$
\frac{1}{p}+\frac{1}{q} \leq \frac{5}{12}
$$

with $q \geq 6$.

Thanks to Lemma 2.3 again, as $\varepsilon$ tends to zero, we have

$$
A \rightarrow 0 \text {. }
$$


An argument similar to that given above in the control for $A$ shows that the terms $B, D, E_{21}$ in $R_{\varepsilon}(t, x)$ here converge to zero, as $\varepsilon \rightarrow 0$, in particular,

$$
B-D-E_{21} \rightarrow 0 \text {. }
$$

Note that $\rho_{t}$ is bounded in $L^{p}\left(0, T ; L^{\frac{2 q}{q+2}}(\Omega)\right)+L^{2}\left(0, T ; L^{2}(\Omega)\right)$ and $\rho$ is bounded in $L^{\infty}(0, T ; \Omega)$, we conclude that $E_{31}, E_{32}$ goes to zero as $\varepsilon$ tends to zero. Similar argument can show that $E \rightarrow 0$ as $\varepsilon$ goes to zero. Thus,

$$
R_{\varepsilon} \rightarrow 0
$$

as $\varepsilon \rightarrow 0$.

We are ready to pass to the limits in (2.10). Letting $\varepsilon$ goes to zero, using (2.11)-(2.13), what we have proved is that in the limit,

$$
-\int_{0}^{T} \int_{\Omega} \psi_{t}\left(\frac{1}{2} \rho|\mathbf{u}|^{2}+\frac{\rho^{\gamma}}{\gamma-1}\right) d x d t+\int_{0}^{T} \int_{\Omega} \psi(t) \rho|\mathbb{D} \mathbf{u}|^{2} d x d t=0
$$

for any test function $\psi \in \mathfrak{D}(0, \infty)$.

The final step is to extend our result (2.17) for the test function $\psi(t) \in \mathfrak{D}(-1, \infty)$. To this end, it is necessary for us to have the continuity of $\rho(t)$ and $(\sqrt{\rho} \mathbf{u})(t)$ in the strong topology at $t=0$. Adopting the similar argument in Vasseur-Yu [24], what we expected can be done.

Here we remark that $\sqrt{\rho} \mathbf{u}$ is bounded in $L^{\infty}\left(0, T ; L^{2}(\Omega)\right)$. Using (1.6) and

$$
\rho_{t}=-\operatorname{div}(\rho \mathbf{u}),
$$

we have

$$
\rho_{t} \in L^{2}\left(0, T ; H^{-1}(\Omega)\right) \text {. }
$$

Note that, $\nabla \sqrt{\rho}$ is bounded in $L^{\infty}\left(0, T ; L^{2}(\Omega)\right)$ and $\rho$ is bounded away from infinity. Thus, the identity

$$
\nabla \rho=2 \sqrt{\rho} \nabla \sqrt{\rho}
$$

gives us

$$
\nabla \rho \in L^{2}\left(0, T ; L^{2}(\Omega)\right)
$$

From (2.18) and (2.19), one obtains

$$
\rho \in C\left([0, T] ; L^{2}(\Omega)\right),
$$

thanks to Theorem 3 on page 287 of book [9].

Using

$$
\sqrt{\rho}_{t}=-\operatorname{div}(\sqrt{\rho} \mathbf{u})+\frac{1}{2} \sqrt{\rho} \operatorname{div} \mathbf{u}
$$

we deduce

$$
\sqrt{\rho}_{t} \in L^{2}\left(0, T ; H^{-1}(\Omega)\right) .
$$

Because $\nabla \sqrt{\rho}$ is bounded in $L^{\infty}\left(0, T ; L^{2}(\Omega)\right)$, then

$$
\sqrt{\rho} \in C\left([0, T] ; L^{2}(\Omega)\right) .
$$


In the meanwhile, we see

$$
\begin{aligned}
& \text { ess } \limsup _{t \rightarrow 0} \int_{\Omega}\left|\sqrt{\rho} \mathbf{u}-\sqrt{\rho_{0}} \mathbf{u}_{0}\right|^{2} d x \\
& \leq \operatorname{ess} \limsup _{t \rightarrow 0}\left(\int_{\Omega}\left(\frac{1}{2} \rho|\mathbf{u}|^{2}+\frac{\rho^{\gamma}}{\gamma-1}\right) d x-\left.\int_{\Omega}\left(\frac{1}{2} \rho_{0}\left|\mathbf{u}_{0}\right|^{2}+\frac{\rho_{0}^{\gamma}}{\gamma-1}\right)\right|^{2} d x\right) \\
& +\operatorname{ess} \limsup _{t \rightarrow 0}\left(2 \int_{\Omega} \sqrt{\rho_{0}} \mathbf{u}_{0}\left(\sqrt{\rho_{0}} \mathbf{u}_{0}-\sqrt{\rho} \mathbf{u}\right) d x+\int_{\Omega}\left(\frac{\rho_{0}^{\gamma}}{\gamma-1}-\frac{\rho^{\gamma}}{\gamma-1}\right)\right) .
\end{aligned}
$$

Using (1.4) and the convexity of $\rho \mapsto \rho^{\gamma}$, we have

$$
\begin{aligned}
& \text { ess } \limsup _{t \rightarrow 0} \int_{\Omega}\left|\sqrt{\rho} \mathbf{u}-\sqrt{\rho_{0}} \mathbf{u}_{0}\right|^{2} d x \\
& \leq 2 \operatorname{ess} \limsup _{t \rightarrow 0} \int_{\Omega} \sqrt{\rho_{0}} \mathbf{u}_{0}\left(\sqrt{\rho_{0}} \mathbf{u}_{0}-\sqrt{\rho} \mathbf{u}\right) d x \\
& =W .
\end{aligned}
$$

To show the continuity of $(\sqrt{\rho} \mathbf{u})(t)$ in the strong topology at $t=0$, we need $W=0$. To this end, we introduce the time evolution of the integral averages

$$
t \in(0, T) \longmapsto \int_{\Omega}(\rho \mathbf{u})(t, x) \cdot \psi(x) d x,
$$

which is defined by

$$
\begin{aligned}
& \frac{d}{d t} \int_{\Omega}(\rho \mathbf{u})(t, x) \cdot \psi(x) d x \\
&=\int_{\Omega} \rho \mathbf{u} \otimes \mathbf{u}: \nabla \psi d x+\int_{\Omega} \rho^{\gamma} \operatorname{div} \psi d x+\int_{\Omega} \rho \mathbb{D} \mathbf{u} \nabla \psi d x
\end{aligned}
$$

where $\psi(x) \in C_{0}^{\infty}(\Omega)$ is a test function. All estimates from (1.4), (1.6) and (1.10) imply (2.24) is continuous function with respect to $t \in[0, T]$. On the other hand, we have

$$
\rho \mathbf{u} \in L^{\infty}\left(0, T ; L^{2}(\Omega)\right)
$$

and hence

$$
\rho \mathbf{u} \in C\left([0, T] ; L_{\text {weak }}^{2}(\Omega)\right)
$$

We consider $W$ as follows

$$
\begin{aligned}
& W=2 \operatorname{ess} \limsup _{t \rightarrow 0} \int_{\Omega} \frac{\sqrt{\rho_{0}} \mathbf{u}_{0}}{\sqrt{\rho}}\left(\sqrt{\rho \rho_{0}} \mathbf{u}_{0}-\rho \mathbf{u}\right) d x \\
& \leq 2 \text { ess } \limsup _{t \rightarrow 0} \int_{\Omega} \frac{\sqrt{\rho_{0}} \mathbf{u}_{0}}{\sqrt{\rho}}\left(\sqrt{\rho \rho_{0}} \mathbf{u}_{0}-\rho_{0} \mathbf{u}_{0}\right) d x+2 \operatorname{ess} \limsup _{t \rightarrow 0} \int_{\Omega} \frac{\sqrt{\rho_{0}} \mathbf{u}_{0}}{\sqrt{\rho}}\left(\rho_{0} \mathbf{u}_{0}-\rho \mathbf{u}\right) d x,
\end{aligned}
$$

where we used (1.6).

Using (2.21) and (2.25) in (2.26), one deduces $W=0$ provided that $\sqrt{\rho_{0}} \mathbf{u}_{0} \in L^{4}(\Omega)$. Thus, we have

which gives us

$$
\operatorname{ess} \limsup _{t \rightarrow 0} \int_{\Omega}\left|\sqrt{\rho} \mathbf{u}-\sqrt{\rho_{0}} \mathbf{u}_{0}\right|^{2} d x=0
$$

$$
\sqrt{\rho} \mathbf{u} \in C\left([0, T] ; L^{2}(\Omega)\right) .
$$


By (2.20) and (2.27), we get

$$
\lim _{\tau \rightarrow 0} \frac{1}{\tau} \int_{0}^{\tau} \int_{\Omega}\left(\frac{1}{2} \rho|\mathbf{u}|^{2}+\frac{\rho^{\gamma}}{\gamma-1}\right) d x d t=\int_{\Omega}\left(\frac{1}{2} \rho_{0}\left|\mathbf{u}_{0}\right|^{2}+\frac{\rho_{0}^{\gamma}}{\gamma-1}\right) d x .
$$

Choosing the following test function for (2.17),

$$
\psi_{\tau}(t)=\psi(t) \quad \text { for } t \geq \tau+\frac{1}{K}, \quad \psi_{\tau}(t)=\frac{t}{\tau} \quad \text { for } t \leq \tau, \quad \psi_{\tau} \text { is a } C^{1} \text { smooth function, }
$$

then we get

$$
\begin{aligned}
& -\int_{\tau+\frac{1}{K}}^{T} \int_{\Omega} \psi_{t}\left(\frac{1}{2} \rho|\mathbf{u}|^{2}+\frac{\rho^{\gamma}}{\gamma-1}\right) d x d t+\int_{0}^{T} \int_{\Omega} \psi_{\tau}(t) \rho|\mathbb{D} \mathbf{u}|^{2} d x d t \\
& -\int_{\tau}^{\tau+\frac{1}{K}} \int_{\Omega}\left(\psi_{\tau}\right)_{t}\left(\frac{1}{2} \rho|\mathbf{u}|^{2}+\frac{\rho^{\gamma}}{\gamma-1}\right) d x d t=\frac{1}{\tau} \int_{0}^{\tau} \int_{\Omega}\left(\frac{1}{2} \rho|\mathbf{u}|^{2}+\frac{\rho^{\gamma}}{\gamma-1}\right) d x d t .
\end{aligned}
$$

Note that,

$$
\begin{aligned}
& \left|\int_{\tau}^{\tau+\frac{1}{K}} \int_{\Omega}\left(\psi_{\tau}\right)_{t}\left(\frac{1}{2} \rho|\mathbf{u}|^{2}+\frac{\rho^{\gamma}}{\gamma-1}\right) d x d t\right| \\
& \leq \frac{C}{K} \int_{\Omega}\left(\frac{1}{2} \rho|\mathbf{u}|^{2}+\frac{\rho^{\gamma}}{\gamma-1}\right) d x \rightarrow 0
\end{aligned}
$$

as $K$ goes to large. Letting $K \rightarrow \infty$, from (2.29) we derive

$$
\begin{aligned}
& -\int_{\tau}^{T} \int_{\Omega} \psi_{t}\left(\frac{1}{2} \rho|\mathbf{u}|^{2}+\frac{\rho^{\gamma}}{\gamma-1}\right) d x d t+\int_{0}^{T} \int_{\Omega} \psi_{\tau}(t) \rho|\mathbb{D} \mathbf{u}|^{2} d x d t \\
& =\frac{1}{\tau} \int_{0}^{\tau} \int_{\Omega}\left(\frac{1}{2} \rho|\mathbf{u}|^{2}+\frac{\rho^{\gamma}}{\gamma-1}\right) d x d t .
\end{aligned}
$$

By (2.28), passing into the limit as $\tau \rightarrow 0$ in (2.30), one obtains

$$
\begin{aligned}
-\int_{0}^{T} \int_{\Omega} \psi_{t}\left(\frac{1}{2} \rho|\mathbf{u}|^{2}+\frac{\rho^{\gamma}}{\gamma-1}\right) d x d t & +\int_{0}^{T} \int_{\Omega} \psi(t) \rho|\mathbb{D} \mathbf{u}|^{2} d x d t \\
& =\int_{\Omega}\left(\frac{1}{2} \rho_{0}\left|\mathbf{u}_{0}\right|^{2}+\frac{\rho_{0}^{\gamma}}{\gamma-1}\right) d x .
\end{aligned}
$$

Taking

$$
\psi(t) \begin{cases}=0 & \text { if } t \leq \tilde{t}-\frac{\epsilon}{2} \\ =\frac{1}{2}+\frac{t-\tilde{t}}{\epsilon} & \text { if } \tilde{t}-\frac{\epsilon}{2} \leq t \leq \tilde{t}+\frac{\epsilon}{2} \\ =1 & \text { if } t \geq \tilde{t}+\frac{\epsilon}{2},\end{cases}
$$

then (2.31) gives for every $\tilde{t} \geq \frac{\epsilon}{2}$,

$$
\begin{aligned}
& \frac{1}{\epsilon} \int_{\tilde{t}-\frac{\epsilon}{2}}^{\tilde{t}+\frac{\epsilon}{2}}\left(\int_{\Omega}\left(\frac{1}{2} \rho|\mathbf{u}|^{2}+\frac{\rho^{\gamma}}{\gamma-1}\right) d x\right) d t+\int_{\tilde{t}-\frac{\epsilon}{2}}^{\tilde{t}+\frac{\epsilon}{2}} \int_{\Omega}\left(\frac{1}{2}+\frac{t-\tilde{t}}{\epsilon}\right) \rho|\mathbb{D} \mathbf{u}|^{2} d x d t \\
& +\int_{\tilde{t}+\frac{\epsilon}{2}}^{T} \int_{\Omega} \rho|\mathbb{D} \mathbf{u}|^{2} d x d t=\int_{\Omega}\left(\frac{1}{2} \rho_{0}\left|\mathbf{u}_{0}\right|^{2}+\frac{\rho_{0}^{\gamma}}{\gamma-1}\right) d x .
\end{aligned}
$$


The second term of left hand side in (2.33) is controlled as follows

$$
\int_{\tilde{t}-\frac{\epsilon}{2}}^{\tilde{t}+\frac{\epsilon}{2}} \int_{\Omega}\left(\frac{1}{2}+\frac{t-\tilde{t}}{\epsilon}\right) \rho|\mathbb{D} \mathbf{u}|^{2} d x d t \leq \int_{\tilde{t}-\frac{\epsilon}{2}}^{\tilde{t}+\frac{\epsilon}{2}} \int_{\Omega} \rho|\mathbb{D} \mathbf{u}|^{2} d x d t \rightarrow 0
$$

as $\epsilon \rightarrow 0$. Thanks to the Lebesgue point Theorem, (2.33) gives us

$$
\int_{\Omega}\left(\frac{1}{2} \rho|\mathbf{u}|^{2}+\frac{\rho^{\gamma}}{\gamma-1}\right) d x+\int_{0}^{T} \int_{\Omega} \rho|\mathbb{D} \mathbf{u}|^{2} d x d t=\int_{\Omega}\left(\frac{1}{2} \rho_{0}\left|\mathbf{u}_{0}\right|^{2}+\frac{\rho_{0}^{\gamma}}{\gamma-1}\right) d x .
$$

This ends our proof of Theorem 1.1.

2.2. Proof of Theorem 1.2. Now, let us to address the proof of Theorem 1.2, We can modify the above proof slightly to show Theorem 1.2. The main advantage of condition $0<\rho \leq \rho(t, x)$ is to have $\nabla \mathbf{u} \in L^{2}\left(0, T ; L^{2}(\Omega)\right)$ in Theorem 1.1. Thus, we can drop this restriction for the constant viscosities case.

The one difference is to show $W=0$ in (2.26). In fact, we have

$$
\begin{aligned}
& W=2 \operatorname{ess} \limsup _{t \rightarrow 0} \int_{\Omega} \mathbf{u}_{0}\left(\rho_{0} \mathbf{u}_{0}-\sqrt{\rho_{0} \rho} \mathbf{u}\right) d x \\
& \leq 2 \operatorname{ess} \limsup _{t \rightarrow 0} \int_{\Omega} \mathbf{u}_{0}\left(\rho_{0} \mathbf{u}_{0}-\rho \mathbf{u}\right) d x+2 \operatorname{ess} \limsup _{t \rightarrow 0} \int_{\Omega} \mathbf{u}_{0}\left(\sqrt{\rho}-\sqrt{\rho_{0}}\right) \sqrt{\rho} \mathbf{u} d x .
\end{aligned}
$$

Applying the similar argument, we can show

$$
\rho \mathbf{u} \in C\left([0, T] ; L_{\text {weak }}^{2}(\Omega)\right), \quad \sqrt{\rho} \in C\left(0, T ; L^{2}(\Omega)\right) .
$$

To show $W=0$, we need $\mathbf{u}_{0} \in L^{k}$ where $\frac{1}{k}+\frac{1}{q} \leq \frac{1}{2}$. Here, we have to mention this is also true for the degenerate viscosity.

After modifying the above one, we are able to follow the same argument to show Theorem 1.2 .

\section{ACKNOWLEDGMENTS}

The author would like to thank his mentor, Alexis Vasseur, for his discussions and for his constant encouragement and guidance.

\section{REFERENCES}

[1] D. Bresch, B. Desjardins, Existence of global weak solutions for 2D viscous shallow water equations and convergence to the quasi-geostrophic model. Comm. Math. Phys., 238 (2003), no.1-3, 211-223.

[2] D. Bresch and B. Desjardins, On the construction of approximate solutions for the 2D viscous shallow water model and for compressible Navier-Stokes models. J. Math. Pures Appl. (9) 86 (2006), no. 4, 362-368.

[3] D. Bresch, B. Desjardins, C.-K. Lin, On some compressible fluid models: Korteweg, lubrication, and shallow water systems. Comm. Partial Differential Equations 28 (2003), no. 3-4, 843-868. 
[4] D. Bresch, B. Desjardins, E. Zatorska,Two-velocity hydrodynamics in fluid mechanics: Part II. Existence of global $\kappa$-entropy solutions to the compressible Navier-Stokes systems with degenerate viscosities. J. Math. Pures Appl. (9) 104 (2015), no. 4, 801-836.

[5] T. Buckmaster, C. De Lellis, P. Isett, and L. Szekelyhidi, Jr. Anomalous dissipation for 1/5-Holder Euler flows. Ann. of Math. (2), 182(1):127-172, 2015.

[6] T. Buckmaster, C. De Lellis, and L. Szekelyhidi, Jr. Dissipative Euler flows with Onsager-critical spatial regularity. Comm. Pure and Appl. Math., 2015.

[7] A. Cheskidov, P. Constantin, S. Friedlander, and R. Shvydkoy. Energy conservation and Onsagers conjecture for the Euler equations. Nonlinearity, 21(6):1233-1252, 2008.

[8] P. Constantin, W. E and E. Titi, Onsager's conjecture on the energy conservation for solutions of Euler's equation. Comm. Math. Phys. 165 (1994), no. 1, 207-209.

[9] L. C. Evans, Partial differential equations. Second edition, Graduate Studies in Mathematics, 19. American Mathematical Society, Providence, RI, 2010.

[10] G. L. Eyink. Energy dissipation without viscosity in ideal hydrodynamics. I. Fourier analysis and local energy transfer. Phys. D, 78(3-4):222-240, 1994.

[11] E. Feireisl, A. Novotný, H. Petzeltová, On the existence of globally defined weak solutions to the Navier-Stokes equations. J. Math. Fluid Mech. 3 (2001), 358-392.

[12] E. Feireisl, Dynamics of viscous compressible fluids. Oxford Lecture Series in Mathematics and its Applications, 26. Oxford Science Publications. The Clarendon Press, Oxford University Press, New York, 2004.

[13] E. Feireisl, P. Gwiazda, A. Swierczewska-Gwiazda, and E. Wiedemann, Regularity and energy conservation for the compressible Euler equations. Arch. Ration. Mech. Anal. 223 (2017), no. 3, 1375-1395.

[14] P. Isett, A Proof of Onsager's Conjecture. Preprint, 2016. arXiv:1608.08301

[15] L. Onsager, Statistical Hydrodynamics. Nuovo Cimento (Supplemento), 6, 279 (1949)

[16] T.M. Leslie, R. Shvydkoy, The energy balance relation for weak solutions of the density-dependent Navier-Stokes equations. Preprint, 2016 arXiv:1602.08527

[17] I. Lacroix-Violet, A. Vasseur, Global weak solutions to the compressible quantum navier-stokes equation and its semi-classical limit. Preprint, 2016. arXiv:1607.06646

[18] J. Li, Z.-X. Xin, Global existence of weak solutions to the barotropic compressible Navier-Stokes flows with degenerate viscosities. 2015, arXiv:1504.06826

[19] P.-L. Lions, Mathematical topics in fluid mechanics. Vol. 1. Incompressible models. Oxford Lecture Series in Mathematics and its Applications, 3. Oxford Science Publications. The Clarendon Press, Oxford University Press, New York, 1996.

[20] P.-L. Lions, Mathematical topics in fluid mechanics. Vol. 2. Compressible models. Oxford Lecture Series in Mathematics and its Applications, 10. Oxford Science Publications. The Clarendon Press, Oxford University Press, New York, 1998.

[21] A. Mellet, A. Vasseur, On the barotropic compressible Navier-Stokes equations. Comm. Partial Differential Equations 32 (2007), no. 1-3, 431-452.

[22] J. Serrin, The initial value problem for the Navier-Stokes equations. 1963 Nonlinear Problems (Proc. Sympos., Madison, Wis., 1962) pp. 69-98 Univ. of Wisconsin Press, Madison, Wis.

[23] M. Shinbrot, The energy equation for the Navier-Stokes system. SIAM J. Math. Anal. 5 (1974), 948954.

[24] A. Vasseur, C. Yu, Existence of global weak solutions for 3D degenerate compressible Navier-Stokes equations. Invent. Math. 206 (2016), no. 3, 935-974.

[25] C.Yu, A new proof of the energy conservation for the Navier-Stokes equations. Preprint, 2016, arXiv:1604.05697

Department of Mathematics, The University of Texas, Austin, Texas 78712.

E-mail address: yucheng@math.utexas.edu 